\title{
Carbon fiber. Obtaining, modification, properties, applications.
}

\author{
C Anna N. Nurmukhametova, ${ }^{1+}$ Artur R. Khamidullin, ${ }^{2}$ and Lubov A. Zenitova ${ }^{2 *}$ \\ ${ }^{1}$ Limited Liability Company «Alabuga-Volokno», Umatex Group. State Corporation «Rosatom». Territory \\ of the SEZ "Alabuga", Sh-2, construction 11/9. Yelabuga district, 423601. Tatarstan Republic. Russia. \\ Phone: +78555753400 ext.6114.E-mail:a.nurmukhametova@umatex.com. \\ ${ }^{2}$ Kazan National Research Technological University. K. Marx St., 68. Kazan, 420015. Tatarstan Republic. \\ Russia.Phone: +7 (843)231-42-51.E-mail:zenit@kstu.ru.
}

Keywords: polyacrylonitrile precursor, carbon fiber, modification.

*Supervising author; ${ }^{+}$Corresponding author

\section{Content}

Introduction

1.1. Obtaining polyacrylonitrile fibers

1.2. Review of scientific and technical information and patent literature on polyacrylonitrile precursor

2.1. Carbon fiber. Properties, purpose and fields of application of carbon plastics.

2.2. Overview of dcientific and technical information and carbon fiber patent literature

2.3. Carbon fiber surface modification

Consequence

\begin{abstract}
The main methods for producing a polyacrylonitrile precursor, methods for producing carbon fiber, its properties, and applications are presented. Patent research in the field of polyacrylonitrile precursor and carbon fiber. Technological problems in the subject area are identified, namely the development of technologies and equipment for producing high-strength carbon fiber, the development of technologies and equipment to reduce the cost of carbon fiber production, the development of technologies for improving the quality of carbon fiber-based composites, and the main ways to solve them are given.

Ways to solve them are developing a technology for producing a polyacrylonitrile precursor for producing high-strength carbon fibers by the wet spinning method, developing a "dry-wet" method for producing polyacrylonitrile, developing high-performance equipment for producing technical polyacrylonitrile precursor in the form of bundles, developing technologies and equipment for efficient regeneration and utilization waste, heat and emissions from the production of carbon fibers, the development of new compositions of precursors and the transition to materials with a higher linear density, optimization of the structure of carbon fiber reinforced plastic to increase strength, the development of technologies and the creation of production of modern types of binders, including the addition of nanoparticles.
\end{abstract}

The main methods for modifying the surface of a carbon fiber that are currently existing are considered.

\section{References}

[1] N.N. Machalaba, A.V. Genis. State and prospects of research and production activities of FGUP (Federal State Unitary Enterprise "VNIISV" (SCIENTIFIC RESEARCH INSTITUTE OF SYNTHETIC FIBER). Chemical fibers. 2011. No.1. P.3-10. (russian)

[2] A.A. Sviridov, V.Y. Varshavsky, A.N. Seleznev and etc. Structural and thermal characteristics of polyacrylonitrile fibres as raw material for production of carbon fibres. Chemical fibers. 2009. No.4. P.14-16. (russian)

[3] P. Morgan. Carbon fibers and their composites. Boca Ration: Taylor\&Francis. 2005. 1153p.

[4] K.E. Perepelkin. Carbon fibres with specific physical and physicochemical properties based on hydrated cellulose and polyacrylonitrile precursors. Chemical fibers. 2002. No.4. P.32-40. (russian)

[5] T.V. Komarova. Carbon fiber: text of lectures. Moscow: D.I. Mendeleev University of Chemical Technology of Russia. 1994. 52p. (russian)

[6] Holding company "Composite" [Electronic resource]. Access mode: http://compozit.su (accessed date: 15.01.2020).

[7] E.M. Aizenstein. The synthetic fibers and threads market in the second half of 2007. Chemical fibers. 2008. No.2. P.3-10. (russian) 
[8] E.M. Aizenstein. The world and Russian markets for chemical fibres and thread in 2007. Chemical fibers. 2008. No.6. P.49-59. (russian)

[9] E.M. Aizenstein. Polyester fibers continue to have a strong lead in the global balance of textile raw materials. Chemical fibers. 2009. No.1. P.5-10. (russian)

[10] E.M. Aizenstein. The world and domestic chemical fiber industry in 2013. Chemical fibers. 2014. No.5. P.3-7. (russian)

[11] E.M. Aizenstein. Polyester fibers in 2012. Chemical fibers. 2014. No.1. P.3-6. (russian)

[12] E.M. Aizenstein. Chemical fibers in 2012 in the world and Russian markets. Chemical fibers. 2013. No.6. P.3-8. (russian)

[13] E.M. Aizenstein. The world production of chemical fibers in 2011. Chemical fibers. 2012. No.3. P.3-7. (russian)

[14] Y.N. Sidorenko. Structural and functional fiber composite materials: tutorial. Tomsk: TSU Publishing House. 2006. 107p. (russian)

[15] R. Eslani Farsani, A. Shokuhfar, A. Sedghi. Процесс изготовления углеродных волокон на основе коммерческих полиакрилонитрильных волокон мокрого формования. Химические волокна. 2006. №5. C.31-33.

[16] N. Yusof, A.F. Ismail. Preparation and characterization of polyacrylonitrile / acrylamide-based activated carbon fibers developed using a solvent-free coagulation process. International Journal of Chemical and Environmental Engineering. 2010. No.1. P.79-84.

[17] Z.A. Rogovin. Fundamentals of chemical fiber chemistry and technology. Moscow: Chemistry. 1974. Vol.2. 344p. (russian)

[18] T.P. Ustinova, N.L. Zaitseva. Polyacrylonitrile (PAN) fibers: technology, properties, application fit: course of lectures. Saratov: Saratov State Technical University. 2002. 40p. (russian)

[19] K.E. Perepelkin. Carbon-chain synthetic fibers. Moscow: Chemistry. 1973. 589p. (russian)

[20] V.Y. Varshavsky. Carbon fibers. Moscow: Federal State Unitary Enterprise "PIK VINITI”. 2007. Edition 2.500p. (russian)

[21] A.T. Kaverov, L.F. Selivanova and etc. Changes in the structure and physical and mechanical properties of polyacrylonitrile fiber during heat treatment. Moscow International Composites Conference: abstracts. Moscow. 1990. P.9. (russian)

[22] A.T. Kaverov. Relation between structure and properties of polyacrylonitrile and carbon fibers. Scientific and technical collected book of M.V. Keldysh.NIITP (Scientific Research Institute of Precision Instruments). 1987. P.62-72. (russian)

[23] A.T. Serkov, G.A. Budnitsky, M.B. Radishevsky and etc. Ways for improving the technology of carbon fiber producing. Chemical fibers. 2003. No.2. P.26-30. (russian)

[24] M.B. Radishevsky, A.T. Serkov, G.A. Budnitsky and etc. Improvement of the production technology.for high-strength and high-modulus carbon fibers. Chemical fibers. 2005. No.5. P.11-15. (russian)

[25] L.A. Zlatoustova. Obtaining of polyacrylonitrile tows for carbon fibers: Dissertation ... candidate of chemical sciences: 05.17.06. Moscow. 2006. 271p. (russian)

[26] N.I. Baurova, V.A. Zorin, V.M. Prikhodko. Evaluation of the influence of carbon fiber structural defects on their sensory properties by electron microscopy scanning methods. Chemical fibers. 2014. No.5. P.13-17. (russian)

[27] Patent 2549075 Russian Federation, International Patent Classification ${ }^{7}$ D01D 5/00, D01F 6/18, B01D 43/00. Method of polymer isolation from solution while forming a pan-precursor to obtain carbon fibers. Kulichikhin Valery Grigorievich, Semakov Alexander Vasilievich, Malkin Alexander Yakovlevich, Skvortsov Ivan Yurievich; patent applicant and patent holder Federal State Budgetary Institution of Science A.V. Topchiev Institute of Petrochemical Synthesis of the Order of the Red Banner of Labor of the Russian Academy of Sciences. No. 2013137256/12; pending 09.08.2013; published 20.04.2015; Bulletin No.11. 10p.

[28] Patent 2541473 Russian Federation, International Patent Classification ${ }^{7}$ D01D 1/02, D01F 6/18. Method for obtaining of a copolymer solution based on acrylonitrile in n-methylmorpholine-n-oxide. Makarov Igor Sergeevich, Golova Lyudmila Konstantinovna, Kuznetsova Lyudmila Kuzminichna, Shlyakhtin Andrey Vladimirovich, Nifantyev Ilya Eduardovich, Kulichikhin Valery Grigorievich; patent applicant and patent holder Federal State Budgetary Institution of Science A.V. Topchiev Institute of Petrochemical Synthesis of the Order of the Red Banner of Labor of the Russian Academy of Sciences. No. 2013126904/04; pending 13.06.2013; published 20.02.2015; Bulletin No.5. 8p.

[29] Patent 2702642 Russian Federation, International Patent Classification ${ }^{7}$ D01F 6/18. Non-woven heatinsulating fire-resistant arc-resistant material. Makhov Sergey Alexandrovich, Mezentseva Elena Viktorovna, Gontar Viktor Anatolyevich, Nazartsev Andrey Andreevich, Ivanov Vladislav Viktorovich; 
CARBON FIBER. OBTAINING, MODIFICATION, PROPERTIES, APPLICATIONS.

patent applicant and patent holder Limited Liability Company "Termopol". No. 2019110895; pending 11.04.2019; published 09.10.2019; Bulletin No.28. 12p.

[30] Patent 2497587 Russian Federation, International Patent Classification ${ }^{7}$ B01J 21/18, B01J 23/40, B01D 71/00, B82B 3/00, C07B 35/04. Method of making membrane catalyst and method of hydrocarbon dehydration using obtained catalyst. Ermilova Margarita Meerovna, Efimov Mikhail Nikolaevich, Zemtsov Lev Mikhailovich, Karpacheva Galina Petrovna, Orekhova Natalya Vsevolodovna, Tereshchenko Gennady Fedorovich; patent applicant and patent holder Federal State Budgetary Institution of Science A.V. Topchiev Institute of Petrochemical Synthesis of the Order of the Red Banner of Labor of the Russian Academy of Sciences. No.2011145690/05; pending 11.11.2011; published 10.11.2013; Bulletin No.31. 17p.

[31] Patent 2647861 Russian Federation, International Patent Classification ${ }^{7} \mathrm{C} 08 \mathrm{~F} 2 / 06, \mathrm{C} 08 \mathrm{~F} 2 / 38, \mathrm{C} 08 \mathrm{~F}$ 220/44, C08F 6/00, D01D 5/06, D01F 9/22. Polyacrylonitrile (PAN) polymers with a low polydispersity index (IPD) and carbon fibers made therefrom. Tan Lungui, Thomas Alan D., Harmon Billy D; patent applicant and patent holder SITEK INDUSTRIES INC. No.2016129965; pending 21.11.2014; published 21.03.2018; Bulletin No.9. 25p.

[32] Patent 206457564 China, International Patent Classification ${ }^{7}$ D01D13/02; D01D5/06. PAN precursor coagulating bath corridor degree of depth gradual change formula adjusting device. Liu Hui; Lian Xinshu, Kong Lingqiang, Leng Xiaogui; patent applicant and patent holder PETROCHINA CO LTD. №201621313428; опубл. 01.09.2017.

[33] Patent 106480515 China, International Patent Classification ${ }^{7}$ D01D1/10, D01F9/12. Filtering device for PAN-based carbon fiber precursor spinning. Meng Fanjun, Meng Zishun; patent applicant and patent holder HARBIN TIANSHUN CHEMICAL TECH DEV CO LTD. № 201610926380; published 08.03.2017.

[34] Patent 104231158 China, International Patent Classification ${ }^{7}$ C08F218/08, C08F220/06, C08F220/44, C08F222/02, C08F4/40, C08F6/06, D01D10/02, D01D5/06, D01F6/40, D01F9/22. Preparation method of PAN precursor for carbon fiber. Ouyang Qin, Chen Yousi; patent applicant and patent holder NINGBO INST MATERIALS TECHNOLOGY \& ENG CAS. No.201310232529; published 24.12.2014.

[35] Patent 101161094 Korea, International Patent Classification ${ }^{7}$ C08F20/44, D01D1/02, D01F6/18, D01F9/22. The method for preparing the dope of pan precursor for carbon fiber. Choi Sheong Hyun, Bang Yun Hyuk, Wang Young Soo; patent applicant and patent holder HYOSUNG CORP. No. 20100138321; published 29.06.2012.

[36] V.Y. Varshavsky, E.P. Mayanov, A.V. Gaberling. Polyacrylonitrile fibers and carbon fibers based thereon as nanostructured materials. Composites and nanostructures. 2009. No.4. P.19-27. (russian)

[37] Ribeiro, Luiz Claudio Pardini, Nilton Pereira Alves, Carlos Alberto Rios Brito Júnior. Polímeros Tan, Lianjiang Investigating the Spinnability in the Dry-Jet Wet Spinning of PAN Precursor Fiber. Lianjiang Tan, Huifang Chen, Ding Pan, Ning Pan. Journal of Applied Polymer Science. 2008. Vol.110. P.1997-2000.

[38] R.F. Ribeiro, L.C. Pardini, N.P. Alves, \& C.A.R. Brito Júnior. Thermal Stabilization study of polyacrylonitrile fiber obtained by extrusion. Polímeros. 2015. Vol.25(6). P.523-530. doi:10.1590/01041428.1938

[39] A.V. Genis, A.V. Kuznetsov. The relationship between the filler activity and the polymer matrix structure with the properties of the fibrous composite material. Plastics. 2016. No.11-12. P.27-32. (russian)

[40] M.Y. Nebratenko, Y.A. Naumova, Y.N. Filatov. Organic solvents and spinning solution properties. Bulletin "Fine Chemical Technologies". 2008. Vol.3. No.3. P.32-39. (russian)

[41] A.N. Ryauzov, V.A. Gruzdev, I.P. Baksheev. Chemical fiber production technology. Moscow: Chemistry. 1980. 448p. (russian)

[42] Y.N. Filatov. Electrospinning of fibrous materials (EPI-process). Moscow: Oil and Gas. 1997. 297p. (russian)

[43] M.S.A. Rahaman, A.F. Ismail, A. Mustafa. A review of heat treatment on polyacrylonitrile fiber. Polymer Degradation and Stability. 2007. Vol.92. P.1421-1432.

[44] L.V. Korchina, N.G. Zubova, T.P. Ustinova. Investigation of the influence of pan-tow modification parameters with sizing compounds on its properties. Bulletin of Saratov State Technical University. 2014. No.2. P.83-86. (russian)

[45] Athirah Eleyas, Sharifah Shahnaz Syed Bakar, Norzilah Binti Abdul Halif, Shuhaida Binti Yahud. The effect of flow rate, concentration, and voltage on diameter of pan precursor fiber by electrospinning technique. Journal of Built Environment, Technology and Engineering. 2017. Vol.2. P.71-78.

[46] Yang Sha, Wei Liu, Yue Li1, Weiyu Cao. Formation Mechanism of Skin-Core Chemical Structure within Stabilized Polyacrylonitrile Monofilaments. Nanoscale Research Letters. 2019. P.1-7. 
A.N. Nurmukhametova, A.R. Khamidullin, and L.A. Zenitova

[47] Yu Wang, Yuanjian Tong, Bowen Zhang, Hua Su, Lianghua Xu. Formation of Surface Morphology in Polyacrylonitrile (PAN) Fibers during Wet-Spinning. Journal of Engineered Fibers and Fabrics. 2018. Vol.2. P.52-57.

[48] S. Simamura. Carbon fibers. Moscow: Mir. 1987. 304p. (russian)

[49] Z.A. Zazulina, T.V. Druzhkina. Fundamentals of chemical fiber technology. Moscow: Chemistry. 1985. 303p. (russian)

[50] E. Fitzer. Carbon fibers and carbon composites. Moscow: Mir. 1988. 336p. (russian)

[51] A.K. Berkovich, V.G. Sergeev, V.A. Medvedev, A.P. Malakho. Synthesis of acrylonitrile-based polymers. PAN and carbon fiber production technology. Tutorial of the Moscow State University. Moscow. 2010. 63p. (russian)

[52] S.V. Ananin, E.S. Ananyeva, V.B. Markin. Composite materials. Tutorial Altai State Technical University. Barnaul. 2007. 94p. (russian)

[53] S.V. Matrenin, B.B. Ovechkin. Composite materials and polymer-based coatings. Tutorial, Tomsk. 2008. 197p. (russian)

[54] Use of composite materials in engineering, ed. B. Naughton. Composite materials. Moscow: Mechanical engineering. 1978. Vol.3. 511p. (russian)

[55] K. Sato, E. Tanaka. Carbon-based composite materials reinforced with carbon fiber. Jidosha gijutsu. 1988. Vol.42. No.6. P.673-678. (russian)

[56] G.I. Kudryavtsev. Reinforcing chemical fibers for composite materials. Moscow: Chemistry. 1992. 328p. (russian)

[57] A.I. Kadantseva, V.A. Tverskoy. Carbon fibers. Tutorial of the Moscow Lomonosov Institut of Fine Chemical Technologies. 2008. 55p. (russian)

[58] Destruction and fatigue. Ed. L. Brautman. Composite materials. Moscow: Mechanical engineering. 1978. Vol.5. 294p. (russian)

[59] Mechanics of composite materials. Ed. J. Sendetski. Composite materials. Moscow: Mir. 1978. Vol.2. 132p. (russian)

[60] Composite materials with a metal matrix. Ed. K. Kreider. Composite materials. Moscow: Mechanical engineering. 1978. Vol.4. 503p. (russian)

[61] Interfaces in metal matrix composites. Ed. Metcalfe. Composite materials. Moscow: Mir. 1978. Vol.1. 438p. (russian)

[62] V.V. Vasiliev, V.D. Protasov, V.V. Bolotin. Composite materials. Hand book. Moscow: Mechanical engineering. 1990. 512p. (russian)

[63] Interfaces in polymer matrix composites. Ed. E. Plueddemann Composite materials. Moscow: Mir. 1978. Vol.6. 275p. (russian)

[64] J.B. Donnet, T.K. Wang, S. Rebouillat, J.C.M. Peng. Carbon fibers, 3rd ed. New York: Marcel Dekker. 1998. P.161-229.

[65] Y.G. Bushuev. Carbon-carbon composite materials. Moscow: Mir. 1994. 128p. (russian)

[66] M.R. Alexander, F.R. Jones. Electrochemical modification of surface properties of carbon fibers. Carbon. 1994. Vol.32. P.785.

[67] S. Kimura, E. Yasuda. Composite materials of the carbon-carbon system. Netsu seri. 1986. Vol.26. No.4. P.284-288. (russian)

[68] M.R. Alexander, F.R. Jones. ToF SIMS and XPS studies of carbon fiber surface during electrolytic oxidation in 17O/18O enriched aqueous electrolytes. Carbon. 1996. Vol.34. P.1093.

[69] Patent 4637925, USA Ultra high strength carbon fiber. Н. Тоһгu, M. Yohji, H. Tomitake. Toray Ind., Inc.. No.854979. pending 23.04.86, published 21.01.87.

[70] S. Kikuchi. Modern developments in the field of carbon-carbon composite materials. Nippon fukugo zaire gakkai shi. 1985. Vol.2. No.2. P.56-64.

[71] E. Theodoridou, A.D. Jannakoudakis. Carbon fibre-supported noble metal catalysts: optimization of fibre pretreatment. Phys. Chem. N.F. 1982. Vol.132. P.175.

[72] Patent 5589055 USA Method for preparing carbon fibers. M. Kobayashi, M. Itoh, Y. Matsuhisa, K. Shimizu. International Patent Classification 6 C 25 F 1/00. Toray Ind., Inc.. No.460917; pending 5.6.95; published 31.12.96.

[73] M. Blazewicz, S. Blazewicz, C. Wajler. Chemically and physically functionalized carbon composites - a prospective material for tissue treatment. Ceram. Int. 1994. Vol.20. P.99-103.

[74] T.V. Komarova. Carbon fibers. Text of lectures. D.I. Mendeleev University of Chemical Technology of Russia 1994. 52p. (russian)

[75] C.U. Jr. Pittman, G.-R. He, B.Wu, S.D. Gardner. Chemical modification of carbon fiber surfaces by nitric acid oxidation followed by reaction with tetra ethylenepentamine. Carbon. 1997. Vol.35. P.317-331. 
CARBON FIBER. OBTAINING, MODIFICATION, PROPERTIES, APPLICATIONS.

[76] W. Zhihong, U. Charles, Jr. Pittman. Reactions of defined oxidized carbon fiber surfaces with model compounds and polyurethane elastomers. Carbon. 1995. Vol.33. P.597-605.

[77] S.K. Eung, H.L. Tae, J.K. Eun, Y. Jin-San. Surface Modification of Carbon Fiber and the Mechanical Properties of the Silicone Rubber. Carbon Fiber Composites. Journal of Applied Polymer Science. 2012. Vol.126. P.410-418.

[78] A.S. Tikhomirov, N.E. Sorokina, V.V. Avdeev. Modification of the carbon fiber surface with nitric acid solutions. Inorganic materials. 2011. No.6. P.684-688. (russian)

[79] F. Severinia, L. Formarob, M. Pegoraroa. Chemical modification of carbon fiber surfaces. Carbon. 2002. No.40. P.735-741.

[80] I.A. Rashkovan. The effect of surface modification on carbon fibers properties and the realization of their strength in unidirectional polymer composites. Autoabstract, Mytishchi. 1993. (russian)

[81] S. Lee, T.R. Kim, A.A. Ogale. Surface and structure modification of carbon nanofibers. Synth. Mater. 2007. Vol.157. P.644-650.

[82] R.J. Dienfendorf, E. Tokorsky. High-performance carbon fibers. Polym. Eng. Sci. 1975. Vol.15. P.150-159.

[83] C.U. Pittman, Jr. Wu, Z. Jiang, et al. Reactivities of amine functions grafted to carbon fiber surface by tetra ethylenepentamine. Designing interfacial bonding. Carbon. 1997. Vol.35. P.929-943.

[84] A.J. Klein. Carbon-carbon composites. Metal prog. 1986. Vol.130. No.5. P.64-68.

[85] D. Lozano-Castello, D. Cazola-Amoros. Usefulness of $\mathrm{CO}_{2}$ adsorption at $273 \mathrm{~K}$ for the characterization of porous carbons. Carbon. 2004. Vol.42. P.1233-1242.

[86] P. Bajaj, A. Dhawan. PAN-based activated carbon fibers: Production, characterization and applications. Indian jour. Of Fibre \& Textile research. 1997. Vol.22. P.222-235.

[87] Application 62149971, Japan. Method for making a high strength carbon fiber. Noriaki T., Tohru H., Tomiso H.; Tore K. pending 25.12.85, published 3.07.87.

[88] R.M. Sinelnikova. Thermoelectric treatment of carbon fibers and technology for making of highstrength carboplastics. Autoabstract, Moscow. 1998. (russian)

[89] Application 6276075 Japan, International Patent Classification ${ }^{4}$ D 06 M 10/00. Method for carbon fiber reinforcement. Uno H., Asai H., Nakano F. Mitsubishi rayon k. k . No.62-7008; pending 14.1.87; published 30.11.87.

[90] M. Fujihira, T. Osa. New challenges in organic electrochemistry. Prog. Batteries Solar Cells. 1979. Vol.2. P.244.

[91] W. Song, C. Zhao-Hui. Influence of heat treatment on physical-chemical properties of PAN based carbon fiber. Ceram. Int. 2006. Vol.32. P.291-295.

[92] Y. Eyiti, S. Kimura. Carbon-carbon composite materials. Tanso. 1987. No.130. P.22-33.

[93] A.V. Afanasyev, L.N. Rabinsky, P.V. Sershak. Experimental determination of deformation and strength characteristics of polymer composite materials. Mechanics of composite materials and structures. 2010. Vol.16. No.2. P.214-222. (russian)

[94] V.I. Solodilov, Y.A. Gorbatkina. Properties of unidirectional carbon plastics based on epoxy resin modified with polysulfone or epoxyurethane oligomer. Mechanics of composite materials and structures. 2008. Vol.14. No.2. P.36-41. (russian)

[95] Patent 2534794 Russian Federation, International Patent Classification ${ }^{7}$ D01F 9/22, D04G 5/00. Method of fibrous PAN material binding during the stages of carbon fiber production thereof. Verbets Dmitry Borisovich, Kartazaeva Zoya Valerievna, Nikolaeva Anastasia Vasilievna, Shcherbakova Tatyana Sergeevna; patent applicant and patent holder Open Joint Stock Company "Scientific research institute of graphite-based construction materials“". No.2013112659/05; pending 21.03.2013; published 10.12.2014; Bulletin No.34. 8p.

[96] Patent 2605973 Russian Federation, International Patent Classification ${ }^{7}$ D01F 9/24, D01F 6/74, C01B 31/02. Fiber is a precursor for carbon fibers, carbon fiber and method for making carbon fiber. Irisawa Toshihira, Hatori Hiroaki, Soneda Yasushi, Kodama Masaya; patent applicant and patent holder NATIONAL INSTITUTE OF ADVANCED INDUSTRIAL SCIENCE AND TECHNOLOGY. No.2015125543; pending 25.11.2013; published 10.01.2017; Bulletin No.1. 26p.

[97] Patent 2694030 Russian Federation, International Patent Classification ${ }^{7}$ C08J 5/06,C08J 5/24, D06M 13/07, C08L 79/08,C08K 7/02. Sized carbon fiber and method for its production. Bulgakov Boris Anatolyevich, Babkin Alexander Vladimirovich, Kepman Alexey Valerievich, Avdeev Victor Vasilievich, Dzhevakov Pavel Borisovich, Korotkov Roman Fedorovich; patent applicant and patent holder Closed Joint Stock Company "Institute of New Carbon Materials and Technologies". No. 2017137965; pending 31.10.2017; published 08.07.2019; Bulletin No.19. 30p.

[98] Patent 2034813 Russian Federation, International Patent Classification ${ }^{7}$ C04B 35/56. Composite material. Emyashev A.V., Kostikov V.I., Kolesnikov S.A.; patent applicant and patent holder State 
Research Institute of graphite-based structural materials. No.5055630/33; pending 11.07.1992; published 10.05.1995.

[99] Patent 2560362 Russian Federation, International Patent Classification ${ }^{7}$ D01F 9/12. High-modulus carbon fiber with a modified surface for reinforcing composites and method for its modification. Nikolai Mikhailovich Chernenko, Dmitry Nikolayevich Chernenko, Natalya Yurievna Beilina, Pavel Gennadievich Elizarov, Anatoly Mikhailovich Borisov, Evgeniya Sergeevna Mashkova, Natalia Nikolaevna Andrianova; patent applicant and patent holder of the Joint Stock Company "Research institute of graphite-based structural materials". "NIIgraphite" No.2014116089/05; pending 23.04.2014; published 20.08.2015; Bulletin No.23. 13p.

[100] Patent 2687939 Russian Federation, International Patent Classification ${ }^{7}$ C04B 35/56. Method for strengthening of carbon fiber reinforced polymer composite materials. Zlobina Irina Vladimirovna, Bekrenev Nikolay Valerievich; patent applicant and patent holder Federal State Budgetary Educational Institution of Higher Education "Yuri Gagarin Saratov State Technical University” №2018124713; pending 05.07.2018; published 16.08.2015; Bulletin No.14. 16p.

[101] Patent 2500697 Russian Federation, International Patent Classification ${ }^{18}$ C08L 23/06, C08J 5/04, C08J $5 / 06$, C08K 3/04, B02C 18/00. Method for making of polymer-based composite materials reinforced with carbon fibers. Chukov Dilyus Irekovich, Kaloshkin Sergey Dmitrievich, Cherdyntsev Viktor Viktorovich, Stepashkin Andrey Alexandrovich, Maksimkin Alexey Valentinovich; patent applicant and patent holder Federal State Autonomous Educational Institution of Higher Professional Education "National research technological university" "MISiS". No.2012126857/05; pending 27.06.2012; published 10.12.2013; Bulletin No.34. 5p.

[102] Patent 2687930 Russian Federation, International Patent Classification ${ }^{7}$ B29C 71/04. Method for strengthening of polymer composite materials reinforced with carbon fiber. Zlobina Irina Vladimirovna, Bekrenev Nikolay Valerievich; patent applicant and patent holder Federal State Budgetary Educational Institution of Higher Education "Yuri Gagarin Saratov State Technical University". №2017142074; pending 01.12.2017; published 16.05.2019; Bulletin No.14. 12p.

[103] Patent 1840615 USSR, International Patent Classification ${ }^{7}$ D01F 9/22. Method for carbon fiber reinforcement. Bondarenko Vladimir Mikhailovich, Shibaeva Galina Andreevna, Azarova Maya Timofeevna, Nazarova Valentina Alexandrovna, Budylina Elena Grigorievna; patent applicant and patent holder Scientific-production association "Khimvolokno". No.3027991/04; pending 19.10.1981; published 27.08.2007; Bulletin No.24. 4p.

[104] Patent 2535797 Russian Federation, International Patent Classification ${ }^{7}$ D01F 9/22, B82B 1/00, D01F 1/10, D01F 11/04. Method for oxidative stabilization of polyacrylonitrile fibers filled with carbon nanoparticles. Salnikova Polina Yurievna, Zhiteneva Daria Alexandrovna, Lysenko Alexander Alexandrovich, Lysenko Vladimir Alexandrovich, Gladunova Olga Igorevna and others; patent applicant and patent holder Federal State Budgetary Educational Institution of Higher Professional Education "St. Petersburg State University of Technology and Design". No.2013119304/05; pending 25.04.2013; published 20.12.2014; Bulletin No.35. 7p.

[105] Patent 2416682 Russian Federation, International Patent Classification ${ }^{7}$ D01F 9/12, D01F 11/16, D01F 9/16, D01F 9/22, D01F 11/10. Method for carbon-containing fiber stabilizing and method for carbon fiber producing. Soboleva Marina Vladimirovna, Usov Vitaly Viktorovich, Shmyrev Vladislav Vasilievich; patent applicant and patent holder Soboleva Marina Vladimirovna, Usov Vitaly Viktorovich. No.2009128759/05; pending 28.07.2009; published 20.04.2011; Bulletin No.11. 8p.

[106] Patent 2534779 Russian Federation, International Patent Classification ${ }^{7}$ D01F 9/22, B82B 1/00, D01F 1/10, D01F 6/18. Method for oxidative stabilization of polyacrylonitrile fibers filled with carbon nanotubes. Salnikova Polina Yurievna, Zhiteneva Daria Alexandrovna, Lysenko Alexander Alexandrovich, Lysenko Vladimir Alexandrovich, Gladunova Olga Igorevna,Yakobuk Anatoly Alekseevich and others; patent applicant and patent holder Federal State Budgetary Educational Institution of Higher Professional Education "St. Petersburg State University of Technology and Design". No.2013121957/05; pending 13.05.2013; published 10.12.2014; Bulletin No.34. 7p.

[107] Patent 2615427 Russian Federation, International Patent Classification ${ }^{7}$ D06M 11/83, C08K 3/04, B82B 1/00. Carbon fiber for composite materials with improved electrical conductivity. Witzel Silke, Wohlmann Bernd, Stusgen Silke; patent applicant and patent holder TOHO TENAX EUROPE GMBH. No.2014127519; pending 06.12.2006; published 04.04.2010; Bulletin No.10. 14p.

[108] Patent 2343235 Russian Federation, International Patent Classification ${ }^{7}$ D01F 9/22, D01F 9/12, D01F 9/14. Method for making of high-strength and high-modulus carbon fiber. Kharitonov Andrey Alekseevich; patent applicant and patent holder Kharitonov Andrey Alekseevich. No.2007130808/04; pending 14.08.2007; published 10.01.2009; Bulletin No.1. 9p. 
[109] Patent 2634598 Russian Federation, International Patent Classification $\overline{7}$ C04B 35/83, C04B 35/528. Carbon-carbon composite materials. Sudyukov Pavel Aleksandrovich, Kaisina Tatiana Vladimirovna, Dokuchaev Andrey Georgievich, Bushuev Vyacheslav Maksimovich; patent applicant and patent holder of the Joint Stock Company "Ural Research Institute of Composite Materials". No.2016134382; pending 22.08.2016; published 01.11.2017; Bulletin No.31. 8p.

[110] Patent 2658858 Russian Federation, International Patent Classification ${ }^{7}$ C04B 35/83. Carbon-carbon composite material and method for making products therefrom. Sudyukov Pavel Alexandrovich, Kaisina Tatiana Vladimirovna, Nekrasov Vadim Alexandrovich, Bushuev Vyacheslav Maksimovich; patent applicant and patent holder of the Joint Stock Company "Ural Research Institute of Composite Materials". No.2016135477; pending 31.08.2016; published 25.06.2018; Bulletin No.18. 8p.

[111] Patent 2631037 Russian Federation, International Patent Classification ${ }^{7}$ D01G 1/00, B26D 1/14. Device for dimensional cutting of carbon fiber. Ozolin Alexander Alexandrovich, Lazarev Mikhail Nikolaevich; patent applicant and patent holder is the Russian Federation, on which behalf the Ministry of Industry and Trade of the Russian Federation acts. No.2015145574; pending 14.06.2016; published 15.09.2017; Bulletin No.26. 10p.

[112] Patent 2568733 Russian Federation, International Patent Classification ${ }^{7}$ C01B 31/02, C04B 35/83, B82B 1/00, B82B 3/00, B82Y 40/00. Carbon-carbon composite material and method for making products therefrom. Nikulin Sergey Mikhailovich, Bushuev Vyacheslav Maksimovich, Rozhkov Alexey Valerievich, Turbina Elena Yurievna; patent applicant and patent holder Open Joint Stock Company "Ural Research Institute of Composite Materials". No.2014115831/05; pending 18.04.2014; published 20.11.2015; Bulletin No.32. 10p.

[113] Patent 2687648 Russian Federation, International Patent Classification ${ }^{7}$ D02J 1/18, D06H 7/00, B23K 26/38. Manual for separation of carbon fiber and mashine for its implementation. Marie Giacomo, Khlebnikov Vladimir Viktorovich, Melamed Leonid Borisovich; patent applicant and patent holder Joint Stock Company "Holding Company "Composite". No.2018123947; pending 02.07.2017; published 15.05.2019; Bulletin No.14. 21p.

[114] Patent 2614679 Russian Federation, International Patent Classification ${ }^{7}$ D06M 15/263, D06M 15/643, D06M 17/00, D06M 17/04, D06M 17/10, D06N 7/00, D06M 15/564. Process for manufacturing carbon fiber fabrics and fabric manufactured with this process. Mazini Attilio; patent applicant and patent holder Automobiles Lamborghini S.P.A. No.2014138134; pending 11.02.2013; published 28.03.2017; Bulletin No.10. 7p.

[115] Patent 2601761 Russian Federation, International Patent Classification ${ }^{7}$ B32B 5/12, B29B 15/08, B32B 5/28, B29C 70/22, B32B 5/26, B32B 7/08, D04B 21/16, D04H 3/10, C08J 5/24. Stitched carbon fiber base material and wet prepreg using same. Tsuchiya Yoshinobu, Kasakawa Hidetoshi, Murata Masahiko, Shigenari Yu, Satoh Hiroyuki; patent applicant and patent holder IHI AEROSPACE CO., LTD., IHI CORPORATION. No.2015105369/05; pending 18.07.2013; published 10.11.2016; Bulletin No.31.26p

[116] Patent 2330906 Russian Federation, International Patent Classification ${ }^{7}$ D01F 9/22. Production method of continuous carbon fiber with heightened modulus. Podkopaev Sergey Alexandrovich, Tyumentsev Vasily Alexandrovich; applicant and patent holder Limited Liability Company "Plant of Carbon and Composite Materials". No.2007110120/04; pending 19.03.2007; published 10.08.2008; Bulletin No.22. $5 \mathrm{p}$.

[117] Patent 2534794 Russian Federation, International Patent Classification ${ }^{7}$ D01F 9/22, D04G 5/00. Method of fibrous PAN material binding during the stages of carbon fiber production thereof. Verbets Dmitry Borisovich, Kartazaeva Zoya Valerievna, Nikolaeva Anastasia Vasilievna, Shcherbakova Tatyana Sergeevna; patent applicant and patent holder Open Joint Stock Company "Scientific research institute of graphite-based construction materials "NIIgrafit". No.2013112659/05; pending 21.03.2013; published 10.12.2014; Bulletin No.34. 8p.

[118] Patent 2042753 Russian Federation, International Patent Classification ${ }^{7}$ D01F 9/14, D01F 9/22, D01F 9/32. Method of polyacrylonitrile yarn oxidation in carbon fiber production and a device for its implementation. Serkov A.T., Budnitsky G.A., Radishevsky M.B., Shishkin P.M .; patent applicant and patent holder Scientific and engineering center "Uglekhimvolokno". No.93018601/05; pending 07.04.1993; published 27.08.2005.

[119] Patent 2578253 Russian Federation, International Patent Classification ${ }^{7}$ C01B 31/02, B82B 3/00, B82Y 40/00. Method for modifying of carbon fibers and carbon nanotubes. Staroverov Nikolay Evgenievich; patent applicant and patent holder Staroverov Nikolay Evgenievich. No.2015106734/05; pending 26.02.2012; published 27.03.2016; Bulletin No.9. 3p

[120] Patent 2400577 Russian Federation, International Patent Classification ${ }^{7}$ D01F 9/22, D01F 9/21, D01F $9 / 12$. Method for production of high modulus fiber made from medium strength carbon fibers. 
Balakhonov Yuri Andreevich, Podkopaev Sergey Alexandrovich, Stepanenko Alexey Anatolyevich, Vinogradov Oleg Vladimirovich; patent applicant and patent holder Limited Liability Company "Plant of Carbon and Composite Materials". No.2008134392/04; pending 21.08.2008; published 27.09.2010; Bulletin No.27. 8p.

[121] Patent 2413799 Russian Federation, International Patent Classification ${ }^{7}$ D01F 9/12. Method for carbon fiber reinforcement. Karpeikin Igor Sergeevich, Sirotkin Oleg Sergeevich, Libkind Evgeniy Azarevich, Kobets Leonid Pavlovich, Trofimova Maria Vladimirovna, Lukyanov Sergey Nikolaevich, Baktenkov Alexander Vasilievich; patent applicant and patent holder Open Joint Stock Company "National Institute of Aviation Technologies". No.2010116839/12; pending 29.04.2010; published 10.03.2011; Bulletin No.7. 8p.

[122] Patent 2127335 Russian Federation, International Patent Classification ${ }^{7}$ D01F 6/40. Method for production of polyacrylonitrile yarns suitable for the production of thin high-strength carbon tapes. Serkov A.T., Matveev V.S., Budnitskiy G.A., Zakharov A.G., Zlatoustova L.A., Kalacheva A.V.; patent applicant and patent holder All-Russian Research Institute of Polymer Fibers with a pilot plant. No.96122271/04; pending 20.11.1996; published 10.03.1999.

[123] Patent 2523483 Russian Federation, International Patent Classification ${ }^{7}$ D01F 11/16, B82B 3/00, C08B 31/02. Method for carbon fiber reinforcement. Urvanov Sergey Alekseevich, Blank Vladimir Davydovich, Khaskov Maxim Aleksandrovich, Karaeva Aida Razim kyzy, Mordkovich Vladimir Zalmanovich, Alshevsky Yuri Lvovich; patent applicant and patent holder Federal state budgetary scientific Institution "Technological institute of superhard and new carbon materials". No.2012155621/05; pending 21.12.2012; published 20.07.2014; Bulletin No.20. 11p.

[124] Patent 2167225 Russian Federation, International Patent Classification ${ }^{7}$ D01F 9/00, D01F 9/22, D01F 9/32. Method for oxidizing of polyacrylonitrile tow and a device for its implementation. Panichkina O.N., Serkov A.A., Serkov A.T., Budnitskiy G.A., Seitova L.N .; patent applicant and patent holder All-Russian Research Institute of Polymer Fibers with a pilot plant. No.99121459/04; pending 11.10.1999; published 20.05.2001; Bulletin No.14.

[125] Patent 10442934 USA, International Patent Classification ${ }^{7}$ C08F120/44, C09C1/48, D01F1/10, D01F9/127, D01F9/22. Methods of Using N-Containing Compounds with Carbon Black to Replace Pan and Form Carbon Fibers. Kemp Richard Alan, Taha Mahmoud Reda; patent applicant and patent holder STC UNM. No.2020002543; pending 16.06.2017; published 15.10.2019. 13p.

[126] Patent 20180126202 Korea, International Patent Classification ${ }^{7}$ D01F9/22; H05K9/00. PAN PANbased carbon fiber whose electro conductive is controlled and method for manufacturing the same. Kim Won Seok, Kim Ji Yeon; patent applicant and patent holder Korea institute of Carbon Convergence Tech. No.20170060903; published 27.11.2018. 13p.

[127] Patent 104630937 China, International Patent Classification ${ }^{7}$ C08F220/14, C08F220/46, C08F222/02, D01D10/06, D01D5/06, D01D5/14, D01F11/12, D01F6/38, D01F9/22. Polyacrylonitrile (PAN)-based high-strength and high-modulus carbon fiber. Zhu Aimin; patent applicant and patent holder NANTONG SUZHONG TEXTILE CO LTD. №20151072166; published 20.05.2015.

[128] Donghwan Cho, Sung Bong Yoon, Chae Wook Cho, Jong Kyoo Park. Effect of additional heat-treatment temperature on chemical, microstructural, mechanical, and electrical properties of commercial PAN-based carbon fiber. Carbon Letters. 2011. Vol.12. No.4. P.223-228.

[129] V.Y. Varshavsky, V.A. Morozov. About graphitizability of polyacrylonitrile fibers based carbon fibers. Composites and nanostructures. 2012. No.1. P.2-8. (russian)

[130] M.A. Kozykina, E.Z. Fainberg, S.P. Papkov, V.Y. Varshavsky, N.P. Radimov, A.A. Konkin. Thermodynamics of organic compounds. Gorky: Technical University of Gorky. 1976. 79p. (russian)

[131] A.A. Konkin. Carbon and other heat-resistant fibers. Moscow: Chemistry. 1974. 376p. (russian)

[132] N.G. Zubova. The structure and properties of composite materials for functional purposes based on epoxy and polyamide matrices and a modified PAN-precursor: Extended abstract of dissertation ... candidate of technical sciences: 05.17.06. Federal state-funded educational institution of higher professional education «Yuri Gagarin State Technical University of Saratov». Saratov. 2012. 21p. (russian)

[133] A.I. Sidorina. Study of the processes of obtaining and thermochemical transformations of polyacrylonitrile nanofibers: Extended abstract of dissertation ... candidate of technical sciences: 05.17.06. Federal state-funded educational institution of higher professional education "Moscow state university of design and technology". Moscow. 2014. 21 p. (russian)

[134] A.A. Gubanov. Development of the process of electrochemical modification of the carbon fiber surface in order to increase the strength of carbon fibre reinforced plastics: Dissertation ... candidate of technical sciences: 05.17.06, 05.17.03. Federal state-funded educational institution of higher 
professional education D. I. Mendeleev University of Chemical Technology of Russia Moscow. 2015. 148 p. (russian)

[135] M.V. Rylkova. Production of fibrous materials based on complexing water-soluble polymers by electrospinning: Dissertation ... candidate of technical sciences: 05.17.06. Moscow State University of Design and Technology. Moscow. 2014. 121p. (russian)

[136] Shamim Zargham, Saeed Bazgir, Ali Asghar Katbab, Abosaeed Rashidi. Influence of KMnO4 concentration and treatment time on PAN precursor and the resulting carbon nanofibers' properties. e-Polymers. 2014. No.14. P.363-372.

[137] Hamid Khayyama, Reza N. Jazara, Srinivas Nunnab, Gelayol Golkarnarenjib, Khashayar Badiib, Seyed Mousa Fakhrhoseinib, Satish Kumarc, Minoo Naebeb. PAN precursor fabrication, applications and thermal stabilization process in carbon fiber production: Experimental and mathematical modelling. Progress in Materials Science. 2020. Vol.107. P.1-39.

[138] Musa Akdere, Felix Pohlkemper, Gunnar Seide, Thomas Gries. Simulation Model for Stabilization of Carbon Fibres. Marmara Journal of Pure and Applied Sciences. 2015. Special Iss.1. P.19-22.

[139] A.T. Mukhamedzyanov, A.A. Mukhamedzyanova, R.N. Gimaev, R.N. Galiakhmetov. State and prospects of production and consumption of carbon fibers made from petroleum pitches. Bulletin of the Bashkir University. 2015. Vol.20. No.4. P.1218-1222. (russian)

[140] L.A. Zemskova, I.V. Sheveleva. Modified sorption-active carbon fiber materials. Russian chemical journal. 2004. Vol.XLVIII. No.5. P.53-57. (russian)

[141] Lokeshwari N., Keshava Joshi, Ashwini Bharadwaj, Anusha Shirkol. PAN based carbon fibre manufacturing. International Journal For Research In Applied And Natural Science. 2016. Vol.2. P.43-52.

[142] Huang Xiaosong. Fabrication and Properties of Carbon Fibers. Materials. 2009. Vol.2. P.2369-2403.

[143] B.S. Kleusov, A.L. Melamed, E.G. Cheblakova. Determination of the heat treatment temperature of pan-precursor-based carbon fiber according to X-ray phase analysis data. Collectied book of abstracts of the Second International conference of young scientists working in the field of carbon materials. 2019. P.141-143. (russian)

[144] A.M. Madoyan, N.A. Popova, N.N. Molotkova. Siliceous polyacrylonitrile - precursors for carbon fibers. Collectied book of abstracts of the Second International conference of young scientists working in the field of carbon materials. 2019. P.181-183. (russian)

[145] V.F. Kablov, N.A. Keibal, V.G. Kochetkov. Investigation of the influence of carbon microfiber on the properties of elastomeric fire and heat-resistant materials. Journal of applied chemistry. 2018. Vol.91. No.7. P.1024-1028. (russian)

[146] S.Y. Voronina, A.Y. Vlasov, V.D. Voronchikhin. Determination of the surface properties of carbon fiber in the process of contact interaction with polymer binders. Journal of applied chemistry. 2018. Vol.91. No.8. P.1148-11153. (russian)

[147] Asma A. Eddib, D.D.L. Chung. Electric permittivity of carbon fiber. Carbon. 2019. Vol.143. P.475-480.

[148] E.A. Sergeeva, I.S. Abdullin, L.A. Zenitova, K.D. Kostina. Analysis of methods for fibrous material modifying. Technological University Bulletin. 2015. Vol.18. No.20. P.164-167. (russian)

[149] Electronic scientific journal "Investigated in Russia" http://zhurnal.ape.relarn.ru/articles/2005/190.pdf / Surface morphology of the original and modified carbon fibers ACTILEN-B according to scanning electron and atomic force microscopy (accessed date 25.01.2020)

[150] S.-J. Park, B.-J. Park, S.-K. Ryn. Carbon. 1999. Vol.37. P.1223.

[151] Z.R. Yue, W. Jang, L. Wang et all. Carbon. 1999. Vol.37. P.1607.

[152] E. Ayranci and B.E. Corway. J. of Applied Electrochemistry. 2001. Vol.31. P.257.

[153] E. Theodoridous, A.D. Jannokoudokis, P.D. Jannokoudakis et all. Can. J. of Chemistry. 1991. Vol.69. P.1881.

[154] C.V. Pittman, H.W. Jiang, Z.R. Yue, at all. Carbon. 1999. Vol.37. P.85.

[155] L.A. Zemskova, I.V. Scheveleva, V.Y. Glushchenko. Sorption properties of activated chitin and chitosan. Chemical technology. 2004. No.7. P.6-11. (russian)

[156] V.B. Fenelopov. Porous carbon. Institute of Catalysis of thr Siberian Branch of the Russian Academy of Sciences, Novosibirsk. 1995. (russian)

[157] A.N. Cherkasov, V.A. Pasechnik. Membranes and sorbents in biotechnology. Chemistry, Leningrad. 1991. 240p. (russian)

[158] A.A. Bukharaev, D.V. Ovchinnikov, A.A. Bukharaeva. Surface diagnostics using a scanning force microscopy (review). Factory laboratory. 1997. No.5. P.10-27. (russian)

[159] R.Z. Bakhtizin. A scanning tunneling microscopy is a new method for studying the surface of bodies. Soros Educational Journal. 2000. Vol.6. No.11. P.1-7. (russian)

[160] Patent 2026732 Russian Federation, International Patent Classification ${ }^{7}$ B01J 20/06. A sorbent obtaining method for extracting of proteins. Vasilevsky V.A., Avramenko V.A., Zemskova L.A., 
Sokolnitskaya T.A. patent applicant and patent holder Institute of Chemistry of the Far Eastern Branch of the Russian Academy of Sciences. No.5009327/26; pending 03.10.1991; published 20.01.1995; Bulletin No.2.

[161] Patent 2075170 Russian Federation, International Patent Classification ${ }^{7}$ B01J 20/06, B01J 20/30. Method of thin-layer inorganic sorbents production. Zemskova L.A., Yakimovich E.L., Avramenko V.A., Zheleznov V.V., Glushchenko V.Y.; patent applicant and patent holder Institute of Chemistry of the Far Eastern Branch of the Russian Academy of Sciences. No. 94026992; pending 18.07.1994; published 1997; Bulletin No.7.

[162] E.S. Ananyeva, S.V. Ananyin. Plasma-chemical modification of carbon fiber surface. Polzunovsky Bulletin. 2009. No.4. P.220-222. (russian)

[163] Protective coatings for carbon fibers (electronic resource) https://www.viam.ru/public/ (accessed date $01 / 25 / 2020)$

[164] A.P. Dostanko, A.O. Korobko, S.M. Zavadsky, N.A. Krekoten. Properties of carbon fibers modified by ion-beam machining. Bulletin of the Polotsk State University. 2009. No.8. P.2-6. (russian)

[165] K.E. Perepelkin. Chemical fibers: present and future. Chemical fibers. 2000. No.5. P.3-17. (russian)

[166] S. Zhang [et al.]. Improving the adhesion of amorphous carbon coatings on cemented carbide through plasma cleaning. Surface and Coatings Technology. 1999. Vol.113. P.120-125.

[167] S.-J. Park [et al.]. Influence of oxygen plasma treatment on impact behaviors of carbon fibers-reinforced composites. Solid State Phenomena. 2007. Vol.119. P.159-162.

[168] L.A. Zemskova. Electrochemical methods of concentration on electrodes made of carbon fiber materials. Chemical technology. 2004. No.7. P.6-11. (russian)

[169] A.A. Berlin. Modern polymer composite materials (PCM). Soros Educational Journal. 1995. No.1. P.57-65. (russian)

[170] R.Z. Bakhtizin. A scanning tunneling microscopy is a new method for studying the surface of bodies. Soros Educational Journal. 2000. Vol.6. No.11. P.1-7. (russian)

[171] I.S. Strakhov, A.A. Gubanov, Y.V. Korshak and etc. Electrochemical treatment of carbon fiber in order to increase the strength of microplastics. Advances in chemistry and chemical technology. 2013. Vol.XXVII. No.7. P.18-21. (russian)

[172] A.R. Garifullin, I.I. Karimullin, A.E. Karnoukhov, M.F. Shaekhov. Investigation of the mechanical properties of carbon fiber-reinforced plastics based on carbon fabrics modified by low-temperature plasma. Technological University Bulletin. 2016. Vol.19. No.22. P.78-80. (russian)

[173] A.R. Garifullin, I.Sh. Abdullin, E.A. Skidchenko. Investigation of the plasma effect on the strength of carbon fiber bond with an epoxy matrix in composite material production. Technological University Bulletin. 2014. Vol.17. No.21. P.69-70. (russian)

[174] A.R. Garifullin, I.S. Abdullin. Investigation of the carbon fibers properties modified by a highfrequency capacitive discharge. Bulletin of Kazan Technological University. 2014. Vol.17. No.18. P.3234. (russian)

[175] L.A. Zemskova. Modified carbon fibers: sorbents, electrode materials, catalysts. Bulletin of the Far Eastern Branch of the Russian Academy of Sciences. 2009. No.2. P.39-52. (russian)

[176] L.A. Zemskova, I.V. Sheveleva. Modified sorption-active carbon fiber materials. Russian chemical journal 2004. Vol.48. No.5. P.53-57. (russian)

[177] L.A. Zemskova, I.V. Sheveleva, N.N. Barinov, T.A. Kaidalova, A.V. Voit, S.V. Zheleznov. Manganese oxide carbon fiber materials. Journal of applied chemistry. 2008. Vol.81. No.7. P.1109-1114. (russian)

[178] L.A. Zemskova, I.V. Sheveleva, A.V. Voyt, V.I. Sergienko, A.V. Plevaka. Sorption materials based on carbon fibers. Sorption and chromatographic processes. 2006. Vol.6. Part.3. P.1169-1174. (russian)

[179] L.A. Zemskova, A.V. Voit, I.V. Sheveleva, L.N. Mironova. Sorption properties of chitosan-carbon fibrous materials. Journal of physical chemistry. 2007. Vol.81. No.10. P.1856-1859. (russian)

[180] L.A. Zemskova, I.V. Sheveleva, A.V. Voyt, T.B. Emelina, V.Y. Glushchenko. Sorption and Electrosorption of $\mathrm{Cu}$ (II) by modified carbon sorbents. Non-ferrous metals. 2007. No.2. P.57-60. (russian)

[181] A.A. Lysenko. Prospects for the development of research and production of carbon fiber sorbents. Chemical fibers. 2007. No.2. P.4-11. (russian)

[182] P.V. Krivoshapkin. Physics and chemistry of surface modification of cellulose, carbon and ceramic materials with nanoscaled metal oxides: Dissertation ... doctor of science in chemistry: 02.00.04; Institute of Chemistry of the Federal Research Center "Komi Scientific Center of the Ural Branch of the Russian Academy of Sciences". Federal state autonomous educational institution for higher education Saint Petersburg National Research University of Information Technologies, Mechanics and Optics. Saint Petersburg. 2019. 301p. (russian) 
CARBON FIBER. OBTAINING, MODIFICATION, PROPERTIES, APPLICATIONS. $1-42$

[183] Roadmap "The use of nanotechnology in the production of carbon fibers and products based thereon" (electronic resource) https://www.rusnano.com/upload/OldNews/Files/33652/current.pdf (accessed date $01 / 25 / 2020$ )

[184] Patent 2560362 Russian Federation, International Patent Classification ${ }^{7}$ D01F 9/12. High-modulus carbon fiber with a modified surface for reinforcing composites and method for its modification.

Chernenko N. M., Chernenko D.N., Beilina N.Y., Elizarov P.G., Borisov A.M., Mashkova E.S.; patent applicant and patent holder Joint Stock Company "State Research Institute of graphite-based structural materials." NIIgrafit ". No.2014116089/05; pending 23.04.2014; published 20.08.2015; Bulletin No.23. $13 \mathrm{p}$.

[185] Patent 2012696 Russian Federation, International Patent Classification ${ }^{7}$ D01F 11/10. Method for carbon fibers surface treatment. Kilin V.S., Kostikov V.I., Demin A.V., Kuzmin A.G., Morozov V.P.; patent applicant and patent holder State Research Institute of graphite-based structural materials. No.91 5006612; pending 17.10.1991; published 20.08.2015; Bulletin No.23. 13p.

[186] Patent 2578283 Russian Federation, International Patent Classification ${ }^{7}$ C01B 31/02, B82B 3/00, B82Y 40/00. Method for modifying carbon fibers and carbon nanotubes. Staroverov N.E.; patent applicant and patent holder Staroverov Nikolay Evgenievich. No.2015106734/05; pending 26.02.2015; published 27.03.2016; Bulletin No.9. 3p

[187] Patent 2538687 Russian Federation, International Patent Classification ${ }^{7}$ G01N 33/44. Method for determining the degree of carbon tows sizing with pitch and machine for its implementation. Malakho A.P., Shornikova O.N., Kalugin D.I., Galiguzov A.A., Kulakov V.V., Avdeev V.V.; patent applicant and patent holder Open Joint Stock Company "Aviation Corporation "Rubin" (JSC "AK "Rubin"). No.2013102000/15; pending 17.01.2013; published 10.01.2015; Bulletin No.1. 8p.

[188] Patent 2475463 Russian Federation, International Patent Classification ${ }^{7}$ C04B 35/83, D01F 9/127, B82B 3/00. Method for modifying the surface of an inorganic fiber, modified fiber and composite material. Tolbin Alexey Yurievich, Kepman A.V., Malakho Artem Petrovich, Kramarenko Evgeny Ivanovich, Kulakov Valery Vasilievich, Avdeev Viktor Vasilievich; patent applicant and patent holder Closed Joint-Stock Company "GrAVIONICS-K". No.2013102000/15; pending 23.11.2011; published 20.02.2013; Bulletin No.5. 13p.

[189] Patent 2402584 Russian Federation, International Patent Classification ${ }^{7}$ C09C 1/44, C09C 3/08. Modified carbon products and their applications. Hampden-Smith Mark J., Caruso James, Atanassova Paolina, Kirlidis Agatagelos; patent applicant and patent holder Cabot Corporation. No.2006136378/05; pending 15.03.20; published 20.01.2010; Bulletin No.5. 46p.

[190] Patent 106867199 China, International Patent Classification ${ }^{7}$ C08K3/04, C08K7/06, C08L63/00. Oriented graphene oxide modification carbon fibre composite material and preparation method thereof. Shen Xiaojun, Dang Ruiqiong, Yang Xiaohui, Nie Huijie, Dang Chenyang, Mao Kajie; patent applicant and patent holder UNIV JIAXING. No.20171083562; pending 16.02.2017; published 20.06.2017. 6p.

[191] Patent 103321035 China, International Patent Classification ${ }^{7}$ D06M10 /06. Surface modification method of carbon fibre plasma grafted graphene oxide. Deng Chao; Jiang Jianjun; Fang Liangchao; Shi Jingwen; Wang Junbiao; patent applicant and patent holder Univ Northwestern Polytechnic. No.201310269829; published 20.09.2013.

[192] Patent 1101348953 China, International Patent Classification ${ }^{7}$ D01F11/06; D01F6/18; D01F9/22. Surface modification method of polyacrylonitrile fiber for producing high performance carbon fibre. Xiao Liang, Jianzhong Yuan; patent applicant and patent holder Univ Northwestern Polytechnic. No.20081046042; published 21.01.2009.

[193] Patent 1250116 China, International Patent Classification ${ }^{7}$ C01B31/08, D01F11/10, D06M10/00, D01F11/10, D06M10/00. Active carbon fiber surface modifying method. Liu Lang, Li Kaixi, Ling Licheng; patent applicant and patent holder Shanxi Coal Chem Inst. No.19981023523; pending 07.10.1998; published 12.04.2000. 6p.

[194] Patent 101412592 China, International Patent Classification ${ }^{7}$ C03C25 /44. Surface modification method for basalt fibre by using plasma treatment and carbon nano-tube coating. Yingchen Zhang, Hongyan Wu, Yiping Qiu; patent applicant and patent holder Univ Donghua. No.200810202626; pending 12.11.2008; published 22.04.2009. 9p. 